on public roads is a common activity in many occupations and industries. Previous New Zealand (NZ) research suggests that work-related driving contributes significantly to the overall burden of work-related fatal injury. This presentation will identify and describe the contribution of work-related driving injuries to fatalities that occurred on a public road in NZ between 2005 and 2014.

Methods Data collection involved: 1) identifying all possible injury deaths in those aged 0-84 years from mortality records using selected external cause of injury codes; 2) linking these to Coronial case files; 3) reviewing and coding work-related cases; and 4) restricting to motor vehicle traffic crashes (MVTC). Frequencies, percentages and rates per 100,000 workers/persons were calculated. The burden of 'bystanders to others' work' and people 'commuting to/from work' was also quantified.

Results A total of 919 work-related MVTC fatalities occurred on public roads (216 workers, 227 commuters, and 476 bystanders). Work contributed to $33 \%$ of transport-related deaths in New Zealand. The Transport sector was the major contributor to work-related MVTC fatalities (42\%) while the Utilities sector had a notably high rate $(8.5$ per 100,000 workers).

Conclusions One third of MVTC fatalities in NZ are workrelated therefore interventions focusing on occupational and general road safety mechanisms could provide beneficial reductions in the national road toll. These data have informed NZ's new road safety strategy, Road to Zero, informing the introduction of a new pillar in work-related driving.

\section{G.004 REMEDIATING DISABILITY SUPPORT WORKERS' PSYCHOSOCIAL SAFETY USING A WORK SAFETY CLIMATE MEASURE}

${ }^{1}$ Neil Kirby*, 'Julia Harries, ${ }^{2}$ Jerry Ford. ${ }^{1}$ The University of Adelaide, Adelaide, Australia; ${ }^{2}$ Flinders University, Bedford Park, Australia

10.1136/injuryprev-2021-safety.26

Background Disability support workers (DSWs) are at high-risk of psychosocial work hazard exposure. This research reports on the follow-up findings from action research undertaken in three consecutive studies over five years to address DSW psychosocial work safety issues. The first study $(n=99)$ showed DSWs experienced poorer outcomes than norm groups on measures of work safety climate, burnout, physical and mental health, and bullying. In a second study $(n=129)$, stakeholder feedback associated with these measures informed the implementation of seven work safety recommendations. Evaluation after 9 months of implementation showed improved trends using the same measures compared to study 1 . A third study investigated whether gains were maintained after 18 months.

Method A follow-up evaluation (study 3, n=138) used the same measures to examine changes overtime and relative to instrument norms.

Results Follow-up findings showed that all health and safety outcomes had continued to improve. There were significant improvements across the three studies in the measures of work safety climate, personal and work-related burnout, witnessed bullying, and mental and physical health. Favourable work safety climate findings were supported by improved incident and workers' compensation statistics for the organisation.
Conclusions Whilst causation cannot be established (e.g., due to the absence of control groups), progressive improvements in psychosocial safety outcomes support the use of psychosocial measures including work safety climate to assist in the selection and evaluation of work safety interventions.

Learning Outcomes The findings supported the use of standardised measures for benchmarking psychosocial aspects of work safety and for evaluating remedial interventions.

\section{$1 G .005$ DRUG-RELATED DISORDER DIAGNOSES AND DEMOGRAPHIC CHARACTERISTICS ASSOCIATED WITH LEGAL INTERVENTION-RELATED INJURIES}

Terry Bunn*, Ashley Bush, Patrick Ward, Madison Liford. University of Kentucky, Lexington, USA

\subsection{6/injuryprev-2021-safety.27}

Background Law enforcement official (LEO) use of force during suspect restraint/apprehension is a serious concern resulting in suspect and LEO injuries. Drug use has been identified as a factor in use-of-force incidents.

Methods This study characterized Kentucky LEO and suspect legal intervention (LI) related injuries and assessed associations between substance use disorder (SUD) diagnoses and demographic characteristics with ICD-10-CM coded LI emergency department (ED) injury visits for years 2016-2018. Logistic regression models examined the most common LEO and suspect injuries.

Results There was a larger proportion of LEO LI hand injury ED visits compared to suspects; there was a larger proportion of suspect LI head injury ED visits compared to LEOs. Among suspect LI ED visits, there were higher odds of head injury ED visits with a SUD (adjusted OR = 1.92), Appalachian county residence (adjusted OR $=1.45$ ), or manhandling LI (adjusted OR = 1.42). No association was found between SUD diagnoses or demographic characteristics and LEO legal intervention hand-related injury $\mathrm{ED}$ visits.

Conclusions When de-escalation techniques fail and LI is required, LEO tactics other than use-of-force are recommended to reduce injuries. Crisis Intervention Team and other trainings may prove beneficial by reducing the need for force; in cases involving SUDs, it is recommended that suspects be referred to treatment.

Learning Outcomes LEOs should employ alternative tactics to reduce officer and suspect use-of-force injuries during LI. Safer options include control techniques, striking zones on suspects, and other tools and body parts when LEO de-escalation training techniques have failed.

\section{E-Posters P1 - Disaster, Emergency Services \& Safe Communities, March 22, 2021}

\section{P1.001 DEVELOPING THE CITY EMERGENCY-HEALTH RESPONSE CAPABILITY (CERC) TOOL}

Agnes Usoro*, Junaid Razzak. Johns Hopkins University, Baltimore, USA

10.1136/injuryprev-2021-safety. 28

Background There is no validated tool to identify gaps in emergency response systems of urban cities in low-and-middle 